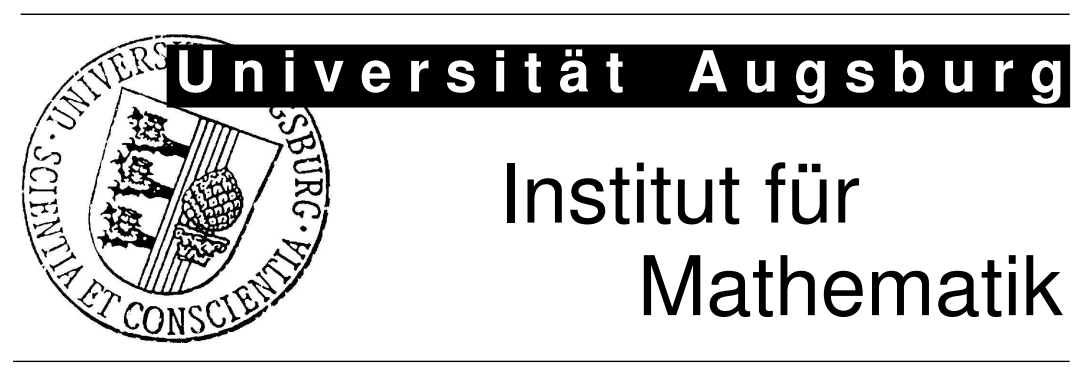

Thomas Franke, Ronald H.W. Hoppe, Christopher Linsenmann, Lothar Schmid, Achim Wixforth

Optimal Control of Surface Acoustic Wave Actuated Sorting of Biological Cells 


\section{Impressum:}

\section{Herausgeber:}

Institut für Mathematik

Universität Augsburg

86135 Augsburg

http://www . math. uni-augsburg.de/de/forschung/preprints.html

\section{ViSdP:}

Ronald H.W. Hoppe

Institut für Mathematik

Universität Augsburg

86135 Augsburg

Preprint: Sämtliche Rechte verbleiben den Autoren (C) 2013 


\title{
Optimal control of surface acoustic wave actuated sorting of biological cells
}

\author{
Thomas Franke, Ronald H.W. Hoppe, Christopher Linsenmann, \\ Lothar Schmid and Achim Wixforth
}

\begin{abstract}
The sorting of biological cells using biological micro-electromechanical systems (BioMEMS) is of utmost importance in various biomedical applications. Here, we consider a new type of devices featuring surface acoustic wave (SAW) actuated cell sorting in microfluidic separation channels. The SAWs are generated by an interdigital transducer (IDT) and manipulate the fluid flow such that cells of different type leave the channel through designated outflow boundaries. The operation of the device can be formulated as an optimal control problem where the objective functional is of tracking type, the state equations describe the fluid-structure interaction between the carrier fluid and the cells, and the control is the electric power applied to the IDT.
\end{abstract}

Mathematics Subject Classification (2010). Primary 65K10 ; Secondary 49M05, 74F10.

Keywords. optimal control, biological cell sorting, surface acoustic waves, finite element immersed boundary method.

\section{Introduction}

We consider the optimal control of surface acoustic wave (SAW) actuated high throughput sorting of biological cells in microfluidic channels which has significant applications in basic cell biology, cancer research, clinical diagnostics, drug design in pharmacology, tissue engineering in reproductive medicine, and transplantation immunology $[3,4,9,13,14]$.

The authors acknowledge support by the German National Science Foundation DFG within the DFG Priority Program SPP 1253 'Optimierung mit partiellen Differentialgleichungen'. The second author also acknowledges partial support by the National Science Foundation NSF (DMS-0914788 and DMS-1115658), the German Federal Ministry for Education and Research BMBF within the collaborative research projects 'FROPT' and 'MeFreSim', and the European Science Foundation ESF within the program 'OPTPDE'.. 
According to [6], the experimental setup consists of a separation channel with three inlets and two outlets. The cells are injected through the middle inlet on the left and can be hydrodynamically focused by the inflows through the other two inlets. SAWs are generated by an Interdigital Transducer (IDT) close to the lateral wall. The IDT features fingers substantially parallel to one another. A static electric field is applied to generate a strain which varies across the aperture of the IDT. The electric field is either perpendicular or parallel to the fingers and created by applying an $\mathrm{AC}$ voltage between two correspondingly positioned conductors. If the IDT is active, the SAWs enter the fluid filled channel and lead to a distortion of the fluid flow that induces acoustic streaming. Let us assume that we have cells of type A and B such that cells of type A should leave the channel through the lower outlet, whereas cells of type B are supposed to leave the channel through the upper outlet. Cells of different type can be distinguished by fluorescence. Without SAW actuation, the inflow velocities are tuned in such a way that a cell of type A leaves through the lower outlet. However, if a cell of type B is detected, the IDT is switched on and the flow is manipulated such that the cell leaves through the upper outlet (cf. Figure 1). In an optimal control setting, the objective is to achieve the sorting as described above, the state equations are given by the fluid-structure interaction between the carrier fluid and the cells, and the control is the time-dependent power applied to the IDT.

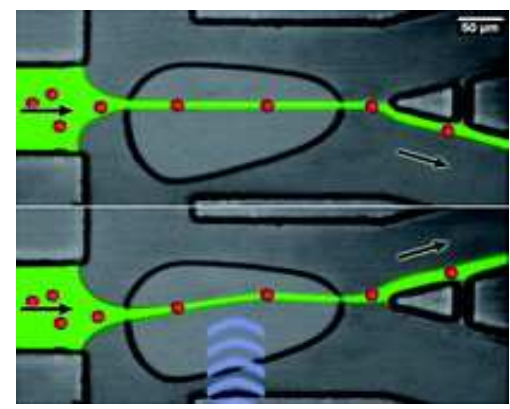

Figure 1. Surface acoustic wave actuated cell sorting (SAWACS) in a microfluidic channel: without SAW actuation (top) and with SAW actuation (bottom).

For the mathematical modeling and numerical simulation of the fluidstructure interaction between the carrier fluid and the cells we will use the finite element immersed boundary (FE-IB) method [1, 2, 8] which is the finite element version of the classical immersed boundary (IB) method originally developed by Peskin (cf., e.g., [11, 12]). The FE-IB method relies on the variational formulation of a coupled system of partial differential equations consisting of the incompressible Navier-Stokes equations and the equations of motion of the boundaries of the immersed cells. As far as the spatial discretization is concerned, we use Taylor-Hood P2/P1 elements for the Navier-Stokes equations and periodic cubic splines for the equations of motion of the immersed 
boundaries. The discretization in time is taken care of by the backward Euler scheme for the semi-discretized Navier-Stokes equations and the forward Euler scheme for the semi-discretized equations of motion. This results in a semiimplicit scheme (Backward Euler/Forward Euler FE-IB method) which has to satisfy a CFL-type condition for stability reasons. We consider a control constrained optimal control problem for the fully discretized FE-IB method featuring an objective functional of tracking type where we prescribe desired positions of the immersed cells. Based on the necessary optimality conditions, the optimal control problem is solved by a projected gradient method with Armijo line search. Numerical results illustrate the performance of the suggested optimal control approach.

\section{The finite element immersed boundary method}

The IB method comprises three groups of equations:

- the Navier-Stokes equations describing the motion of the incompressible viscous carrier fluid,

- the material elasticity equations responsible for the total elastic energy and the resulting forces exerted by the immersed cells,

- the interaction equations translating Eulerian into Lagrangian quantities and vice versa.

We denote by $\Omega \subset \mathbb{R}^{2}$ the Eulerian domain representing the separation channel wit boundary $\Gamma=\bar{\Gamma}_{\mathrm{D}} \cup \bar{\Gamma}_{\mathrm{N}}, \Gamma_{\mathrm{D}} \cap \Gamma_{\mathrm{N}}=\emptyset$, and by $\mathbf{v}(x, t), p(x, t)$ the velocity and the pressure of the carrier fluid in $(x, t) \in \bar{\Omega} \times[0, T], T>0$. We further refer to $\Lambda=[0, L] \subset \mathbb{R}$ as the Lagrangian domain such that the vector valued function $\mathbf{X}(\lambda, t), \lambda \in \Lambda$, represents the closed, non self-intersecting boundary of an immersed cell at time $t \in[0, T], T>0$.

The classical formulation of the IB equations then reads as follows: Find a triple $(\mathbf{v}, p, \mathbf{X})$ such that the incompressible Navier-Stokes equations

$$
\begin{aligned}
\rho\left(\frac{\partial \mathbf{v}}{\partial t}+(\mathbf{v} \cdot \nabla) \mathbf{v}\right)-2 \eta \nabla \cdot \mathbf{D}(\mathbf{v})+\nabla p & =\mathbf{f}_{E} & & \text { in } \Omega \times(0, T] \\
\nabla \cdot \mathbf{v} & =0 & & \text { in } \Omega \times(0, T] \\
\mathbf{v} & =\mathbf{v}_{\mathrm{D}} & & \text { on } \Gamma_{\mathrm{D}} \times(0, T] \\
(-p \mathbf{I}+2 \eta \mathbf{D}(\mathbf{v})) \boldsymbol{\nu} & =\mathbf{0} & & \text { on } \Gamma_{\mathrm{N}} \times(0, T] \\
\mathbf{v}(\cdot, 0) & =\mathbf{v}_{0} & & \text { in } \Omega
\end{aligned}
$$

are satisfied. Here, $\rho$ and $\eta$ are the density and viscosity of the carrier fluid, $\mathbf{D}(\mathbf{v})$ stands for the rate of deformation tensor $\mathbf{D}(\mathbf{v})=\left(\nabla \mathbf{v}+(\nabla \mathbf{v})^{T}\right) / 2, \mathbf{f}_{E}$ is a source term that will be specified in $(2.3 \mathrm{a})$ below, $\mathbf{v}_{\mathrm{D}}$ is a prescribed velocity, $\boldsymbol{\nu}$ denotes the exterior unit normal vector on the Neumann boundary $\Gamma_{\mathrm{N}}$, and $\mathbf{v}_{0}$ refers to the initial velocity. The Navier-Stokes equations are coupled with 
4 T. Franke, R.H.W. Hoppe, C. Linsenmann, L. Schmid and A. Wixforth

the equations of motion of the immersed boundary

$$
\begin{aligned}
\frac{\partial \mathbf{X}}{\partial t}(\lambda, t) & =\mathbf{v}(\mathbf{X}(\lambda, t), t)=\int_{\Omega} \mathbf{v}(\mathbf{x}, t) \cdot \boldsymbol{\delta}(\mathbf{X}(\lambda, t)-\mathbf{x}) \mathrm{d} \mathbf{x} \\
\mathbf{X}(\lambda, 0) & =\mathbf{X}_{0}(\lambda)
\end{aligned}
$$

where $\boldsymbol{\delta}$ stands for the Dirac delta function and $\mathbf{X}_{0}$ is the initial configuration of the immersed boundary. The source term $\mathbf{f}_{E}$ in (2.1a) is a global force density according to

$$
\begin{aligned}
\mathbf{f}_{E}(\mathbf{x}, t) & =\int_{\Lambda} \mathbf{F}_{L}(\lambda, t) \cdot \boldsymbol{\delta}(\mathbf{X}(\lambda, t)-\mathbf{x}) \mathrm{d} \lambda, \\
\mathbf{F}_{L}(\lambda, t) & =-E^{\prime}(\mathbf{X}(\cdot, t))(\lambda),
\end{aligned}
$$

where $E^{\prime}$ is the variational derivative of the elastic energy of the immersed boundary as given by

$$
E(t):=E(\mathbf{X}(\cdot, t)):=\int_{\Lambda} \mathcal{E}^{\mathrm{e}}\left(\frac{\partial \mathbf{X}(\lambda, t)}{\partial \lambda}\right) \mathrm{d} \lambda+\int_{\Lambda} \mathcal{E}^{\mathrm{b}}\left(\frac{\partial^{2} \mathbf{X}(\lambda, t)}{\partial \lambda^{2}}\right) \mathrm{d} \lambda
$$

Here, $\mathcal{E}^{\mathrm{e}}$ and $\mathcal{E}^{\mathrm{b}}$ stand for the local energy densities

$$
\begin{aligned}
\mathcal{E}^{\mathrm{e}}\left(\frac{\partial \mathbf{X}(\lambda, t)}{\partial \lambda}\right) & =\frac{\kappa_{\mathrm{e}}}{2}\left(\left|\frac{\partial \mathbf{X}}{\partial \lambda}(\lambda, t)\right|^{2}-1\right), \\
\mathcal{E}^{\mathrm{b}}\left(\frac{\partial^{2} \mathbf{X}(\lambda, t)}{\partial \lambda^{2}}\right) & =\frac{\kappa_{\mathrm{b}}}{2}\left|\frac{\partial^{2} \mathbf{X}}{\partial \lambda^{2}}(\lambda, t)\right|^{2},
\end{aligned}
$$

with $\kappa_{\mathrm{e}}>0$ and $\kappa_{\mathrm{b}}>0$ denoting the elasticity coefficients for elongationcompression and bending.

The FE-IB method relies on the variational formulation of the coupled system. We introduce the function spaces

$$
\begin{aligned}
\mathbf{V}(0, T) & :=\mathbf{H}^{1}\left((0, T), \mathbf{H}^{-1}(\Omega)\right) \cap \mathbf{L}^{2}\left((0, T), \mathbf{H}^{1}(\Omega)\right), \\
\mathbf{W}(0, T) & :=\left\{\mathbf{v} \in \mathbf{V}(0, T)|\mathbf{v}|_{\Gamma_{\mathrm{D}} \times(0, T)}=\mathbf{v}_{\mathrm{D}}\right\}, \\
Q(0, T) & :=L^{2}\left((0, T), L^{2}(\Omega)\right),
\end{aligned}
$$

and

$$
\begin{aligned}
& \mathbf{X}(0, T):=\mathbf{H}^{1}\left((0, T), \mathbf{L}^{2}(\Lambda)\right) \cap \mathbf{L}^{2}\left((0, T), \mathbf{H}_{\text {per }}^{3}(\Lambda)\right), \\
& \mathbf{H}_{\text {per }}^{3}(\Lambda):=\left\{\mathbf{Y} \in \mathbf{H}^{3}(\Lambda) \mid \partial^{k} \mathbf{Y}(0) / \partial \lambda^{k}=\partial^{k} \mathbf{Y}(L) / \partial \lambda^{k}, k=0,1,2\right\}
\end{aligned}
$$

The FE-IB method amounts to the computation of a triple

$$
(\mathbf{v}, p, \mathbf{X}) \in \mathbf{W}(0, T) \times Q(0, T) \times \mathbf{X}(0, T)
$$


such that for almost all $t \in[0, T]$ and all test functions $(\mathbf{w}, q, \mathbf{Y}) \in \mathbf{H}_{\Gamma_{\mathrm{D}}, 0}^{1}(\Omega) \times$ $L^{2}(\Omega) \times \mathbf{H}_{\text {per }}^{3}(\Lambda)$ it holds

$$
\begin{aligned}
\left\langle\frac{\partial \mathbf{v}}{\partial t}, \mathbf{w}\right\rangle_{\mathbf{H}^{-1}, \mathbf{H}_{\Gamma_{\mathrm{D}}}^{1}, 0}+a(\mathbf{v}, \mathbf{w})-b(\mathbf{w}, p) & =\ell(\mathbf{w}), \\
b(\mathbf{v}, q) & =0 \\
\mathbf{v}(\cdot, 0) & =\mathbf{v}_{0}, \\
\left(\frac{\partial \mathbf{X}}{\partial t}, \mathbf{Y}\right)_{0, \Lambda}-\int_{\Lambda} \mathbf{v}(\mathbf{X}(\lambda, t), t) \cdot \mathbf{Y}(\lambda) \mathrm{d} \lambda & =0 \\
\mathbf{X}(\cdot, 0) & =\mathbf{X}_{0},
\end{aligned}
$$

where $\langle\cdot, \cdot\rangle_{\mathbf{H}^{-1}, \mathbf{H}_{0}^{1}}$ stands for the dual pairing between $\mathbf{H}_{0}^{1}(\Omega)$ and $\mathbf{H}_{\Gamma_{\mathrm{D}}, 0}^{-1}(\Omega)$ and $a(\cdot, \cdot), b(\cdot, \cdot)$, as well as the functional $\ell(\cdot)$ are given by

$$
\begin{aligned}
a(\mathbf{v}, \mathbf{w}) & :=(\rho(\mathbf{v} \cdot \nabla) \mathbf{v}, \mathbf{w})_{0, \Omega}+(\eta \nabla \mathbf{v}, \nabla \mathbf{w})_{0, \Omega} \\
b(p, \mathbf{v}) & :=(p, \nabla \cdot \mathbf{v})_{0, \Omega} \quad, \quad \ell(\mathbf{w}):=\left\langle\mathbf{F}_{L}, \mathbf{w}\right\rangle_{\mathbf{H}^{-1}, \mathbf{H}_{0}^{1}} .
\end{aligned}
$$

For the numerical solution of (2.5) we use Taylor-Hood P2/P1 elements for the spatial discretization of (2.5a)-(2.5c) with respect to a quasi-uniform simplicial triangulation $\mathcal{T}_{h}(\Omega)$ of $\Omega$ that aligns with the partition of $\Gamma$ and periodic cubic splines for the spatial discretization of $(2.5 \mathrm{~d}),(2.5 \mathrm{e})$ with respect to an equidistant partition

$$
\mathcal{T}_{\Delta \lambda}(\Lambda):=\left\{0=\lambda_{0}<\lambda_{1}<\cdots<\lambda_{R}=L\right\}
$$

of $\Lambda$ into subintervals $\Lambda_{r}:=\left[\lambda_{r-1}, \lambda_{r}\right], 1 \leq r \leq R$, of length $\Delta \lambda=L / R$. We note that the discrete immersed cell occupies subdomains $B_{\Delta \lambda, t} \subset \Omega$ with boundaries $\partial B_{\Delta \lambda, t}$ that are $\mathbf{C}^{2}$ curves described by the periodic cubic spline. We introduce the finite element spaces

$$
\begin{aligned}
\mathbf{V}_{h} & :=\left\{\mathbf{v} \in \mathbf{C}^{0}(\Omega)|\mathbf{v}|_{T} \in \mathbf{P}_{2}(T), T \in \mathcal{T}_{h}(\Omega)\right\} \\
\mathbf{V}_{\Gamma_{\mathrm{D}}, h} & :=\left\{\mathbf{v}_{h} \in \mathbf{V}_{h}\left|\mathbf{v}_{h}\right|_{\Gamma_{\mathrm{D}}}=\mathbf{v}_{h, D}\right\} \\
\mathbf{V}_{0, h} & :=\left\{\mathbf{v}_{h} \in \mathbf{V}_{h}\left|\mathbf{v}_{h}\right|_{\Gamma_{\mathrm{D}}}=\mathbf{0}\right\} \\
Q_{h} & :=\left\{q \in L^{2}(\Omega)|q|_{T} \in P_{1}(T), T \in \mathcal{T}_{h}(\Omega)\right\},
\end{aligned}
$$

where $\mathbf{v}_{h, D}$ is a piecewise polynomial approximation of $\mathbf{v}_{\mathrm{D}}$, and

$$
\begin{aligned}
\mathbf{S}_{\Delta \lambda}:=\left\{\mathbf{X}_{\Delta \lambda} \in \mathbf{C}^{2}(\Lambda)\left|\mathbf{X}_{\Delta \lambda}\right|_{\Lambda_{r}} \in \mathbf{P}_{3}\left(\Lambda_{r}\right), 1 \leq r \leq R,\right. \\
\\
\left.\mathrm{d}^{k} \mathbf{X}_{\Delta \lambda} / \mathrm{d} \lambda^{k}\left(\lambda_{0}\right)=\mathrm{d}^{k} \mathbf{X}_{\Delta \lambda} / \mathrm{d} \lambda^{k}\left(\lambda_{R}\right), k=0,1,2\right\} .
\end{aligned}
$$

The semi-discretization of (2.5) in space requires the computation of a triple

$$
\left(\mathbf{v}_{h}, p_{h}, \mathbf{X}_{\Delta \lambda}\right) \in \mathbf{C}^{1}\left((0, T), \mathbf{V}_{\Gamma_{\mathrm{D}}, h}\right) \times L^{2}\left((0, T), Q_{h}\right) \times \mathbf{C}^{1}\left((0, T), \mathbf{S}_{\Delta \lambda}\right)
$$


such that for all $t \in[0, T]$ and all test functions $\mathbf{w}_{h} \in \mathbf{V}_{0, h}, q_{h} \in Q_{h}$, and $\mathbf{Y}_{\Delta \lambda} \in \mathbf{S}_{\Delta \lambda}$ it holds

$$
\begin{aligned}
\left(\frac{\partial \mathbf{v}_{h}}{\partial t}, \mathbf{w}_{h}\right)_{0, \Omega}+a\left(\mathbf{v}_{h}, \mathbf{w}_{h}\right)-b\left(\mathbf{w}_{h}, p_{h}\right) & =\ell\left(\mathbf{w}_{h}\right) \\
b\left(\mathbf{v}_{h}, q_{h}\right) & =0 \\
\mathbf{v}_{h}(\cdot, 0) & =\Pi_{h} \mathbf{v}_{0} \\
\left(\frac{\partial \mathbf{X}_{\Delta \lambda}}{\partial t}, \mathbf{Y}_{\Delta \lambda}\right)_{0, \Lambda}-\int_{\Lambda} \mathbf{v}_{h}\left(\mathbf{X}_{\Delta \lambda}(\lambda, t), t\right) \cdot \mathbf{Y}_{\Delta \lambda}(\lambda) \mathrm{d} \lambda & =0 \\
\mathbf{X}_{\Delta \lambda}(\cdot, 0) & =\Pi_{\Delta \lambda} \mathbf{X}_{0}
\end{aligned}
$$

where $\Pi_{h}$ and $\Pi_{\Delta \lambda}$ are the $L^{2}$-projections onto $\mathbf{V}_{h}$ and $\mathbf{S}_{\Delta \lambda}$, respectively. For the algebraic formulation of (2.7) we equip $\mathbf{V}_{0, h}, Q_{h}$, and $\mathbf{S}_{\Delta \lambda}$ with canonical bases $\left\{\boldsymbol{\phi}_{i}\right\}_{i=1}^{N_{1}},\left\{\psi_{i}\right\}_{i=1}^{N_{2}}$, and $\left\{\mathbf{B}_{i}\right\}_{i=1}^{N_{3}}$. Accordingly, we write

$$
\mathbf{v}_{h}=\sum_{i=1}^{N_{1}} v_{i} \phi_{i}, \quad p_{h}=\sum_{i=1}^{N_{2}} p_{i} \psi_{i}, \quad \mathbf{X}_{\Delta \lambda}=\sum_{i=1}^{N_{3}} X_{i} \mathbf{B}_{i}
$$

Here, the $\mathbf{B}_{i}$ are the B-splines with respect to the partition $\mathcal{T}_{\Delta \lambda}(\Lambda)$ and $X_{1}, \ldots, X_{N_{3}}$ are the de Boor points. As an important assumption we state that the Lagrangian force density $\mathbf{F}_{L}$ gets discretized by means of $\left\{\mathbf{B}_{i}\right\}$ as well in order to gain a useful transpose property (see (2.8a) below). Furthermore, we denote by $M_{L}$ and $M_{E}$ the Lagrangian and the Eulerian mass matrix, respectively, by $C(v)$ the advection matrix, by $A$ the stiffness matrix, by $B$ the matrix associated with the divergence operator, and by $K(X) \in \mathbb{R}^{N_{1} \times N_{3}}$ the matrix with components $\int_{\Lambda} \phi_{i}\left(\mathbf{X}_{\Delta \lambda}(\lambda)\right) \cdot \mathbf{B}_{j}(\lambda) \mathrm{d} \lambda$. We assume that all (Eulerian) matrices and right-hand sides are manipulated appropriately in order to enforce the Dirichlet conditions from (2.1c). Then the algebraic formulation of (2.7) reads: Find $(v, p, X):[0, T] \rightarrow \mathbb{R}^{N_{1}} \times \mathbb{R}^{N_{2}} \times \mathbb{R}^{N_{3}}$, such that for almost all $t \in[0, T]$

$$
\begin{aligned}
M_{E} \frac{\mathrm{d} v}{\mathrm{~d} t}(t)+C(v(t)) v(t)+A v(t)+B^{\top} p(t) & =K(X(t))^{\top} F_{L}(X(t)) \\
B v(t) & =0 \\
\sum_{i=1}^{N_{1}} v_{i}(0) \boldsymbol{\phi}_{i} & =\Pi_{h} \mathbf{v}_{0} \\
M_{L} \frac{\mathrm{d} X}{\mathrm{~d} t}(t) & =K(X(t)) v(t), \\
\sum_{i=1}^{N_{3}} X_{i}(0) \mathbf{B}_{i} & =\Pi_{\Delta \lambda} \mathbf{X}_{0} .
\end{aligned}
$$




\section{The semi-implicit Backward Euler/Forward Euler FE-IB method}

For the discretization in time we first consider the Backward Euler/Forward Euler FE-IB method from [8] in the sense that we discretize the Navier-Stokes equations by the backward Euler method in time and the equation of motion of the immersed boundary by the forward Euler scheme. In particular, we consider an equidistant partition

$$
\mathcal{T}_{\Delta t}:=\left\{0=: t_{0}<t_{1}<\cdots<t_{\mathcal{M}}:=T\right\}, \mathcal{M} \in \mathbb{N},
$$

of the time interval $[0, T]$ into subintervals of length $\Delta t:=T / \mathcal{M}$ and set

$$
\mathbf{v}_{h}^{(m)}:=\mathbf{v}_{h}\left(\cdot, t_{m}\right), \quad p_{h}^{(m)}:=p_{h}\left(\cdot, t_{m}\right), \quad \mathbf{X}_{\Delta \lambda}^{(m)}:=\mathbf{X}_{\Delta \lambda}\left(\cdot, t_{m}\right) .
$$

We refer to

$$
\mathbf{D}_{\Delta t}^{+} \mathbf{v}_{h}^{(m)}:=\left(\mathbf{v}_{h}^{(m+1)}-\mathbf{v}_{h}^{(m)}\right) / \Delta t, \quad \mathbf{D}_{\Delta t}^{-} \mathbf{v}_{h}^{(m)}:=\left(\mathbf{v}_{h}^{(m)}-\mathbf{v}_{h}^{(m-1)}\right) / \Delta t
$$

as the forward and backward difference operator. We further define the total discrete energy by means of

$$
E_{\Delta \lambda}\left(t_{m}\right):=E_{\Delta \lambda}^{\mathrm{e}}\left(t_{m}\right)+E_{\Delta \lambda}^{\mathrm{b}}\left(t_{m}\right),
$$

where the discrete elastic energy $E_{\Delta \lambda}^{\mathrm{e}}\left(t_{m}\right)$ and the discrete bending energy $E_{\Delta \lambda}^{\mathrm{b}}\left(t_{m}\right)$ are given by

$$
\begin{aligned}
& E_{\Delta \lambda}^{\mathrm{e}}\left(t_{m}\right)=\frac{\kappa_{\mathrm{e}}}{2} \int_{\Lambda}\left(\left|\frac{\partial \mathbf{X}_{\Delta \lambda}^{(m)}}{\partial \lambda}(\lambda)\right|^{2}-1\right) \mathrm{d} \lambda \\
& E_{\Delta \lambda}^{\mathrm{b}}\left(t_{m}\right)=\frac{\kappa_{\mathrm{b}}}{2} \sum_{r=1}^{R} \int_{\Lambda_{r}}\left|\frac{\partial^{2} \mathbf{X}_{\Delta \lambda}^{(m)}}{\partial \lambda^{2}}(\lambda)\right|^{2} \mathrm{~d} \lambda .
\end{aligned}
$$

Observing that $\partial^{3} \mathbf{X}_{\Delta \lambda}^{(m)}(\lambda) / \partial \lambda^{3}$ is constant on $\Lambda_{r}$, the discrete force density takes the form

$$
\begin{array}{r}
\left(\mathbf{F}_{L, \Delta \lambda}^{(m)}, \mathbf{w}_{h}\left(\mathbf{X}_{\Delta \lambda}^{(m)}\right)\right)_{0, \Lambda}=-\kappa_{\mathrm{e}} \int_{\Lambda} \frac{\partial \mathbf{X}_{\Delta \lambda}^{(m)}(\lambda)}{\partial \lambda} \cdot \nabla \mathbf{w}_{h}\left(\mathbf{X}_{\Delta \lambda}^{(m)}(\lambda)\right) \frac{\partial \mathbf{X}_{\Delta \lambda}^{(m)}}{\partial \lambda} \mathrm{d} \lambda \\
+\left.\kappa_{\mathrm{b}} \sum_{r=1}^{R} \frac{\partial^{3} \mathbf{X}_{\Delta \lambda}^{(m)}}{\partial \lambda^{3}}\right|_{\Lambda_{r}} \cdot \int_{\Lambda_{r}} \nabla \mathbf{w}_{h}\left(\mathbf{X}_{\Delta \lambda}^{(m)}(\lambda)\right) \frac{\partial \mathbf{X}_{\Delta \lambda}^{(m)}}{\partial \lambda} \mathrm{d} \lambda .
\end{array}
$$

The Backward Euler/Forward Euler FE-IB reads as follows:

Given $\mathbf{v}_{h}^{(0)}=\Pi_{h} \mathbf{v}_{0}$ and $\mathbf{X}_{0, \Delta \lambda}=\mathbf{X}_{\Delta \lambda}^{(0)}=\Pi_{\Delta \lambda} \mathbf{X}_{0}$, for $m=0, \ldots, \mathcal{M}-1$ we perform the following two steps:

Algorithm 3.1 .

(i) Compute $\left(\mathbf{v}_{h}^{(m+1)}, p_{h}^{(m+1)}\right) \in \mathbf{V}_{h, \Gamma_{\mathrm{D}}} \times Q_{h}$ such that for all $\mathbf{w}_{h} \in \mathbf{V}_{h, 0}$

$$
\begin{aligned}
\left(\rho \mathbf{D}_{\Delta t}^{+} \mathbf{v}_{h}^{(m)}, \mathbf{w}_{h}\right)_{0, \Omega}+a\left(\mathbf{v}_{h}^{(m+1)}, \mathbf{w}_{h}\right)-b\left(p_{h}^{(m+1)}, \mathbf{w}_{h}\right) & =\ell_{h}^{(m)}\left(\mathbf{w}_{h}\right), \\
b\left(w_{h}, \mathbf{v}_{h}^{(m+1)}\right) & =0,
\end{aligned}
$$

where $\ell_{h}^{(m)}\left(\mathbf{w}_{h}\right):=\left(\mathbf{F}_{L, \Delta \lambda}, \mathbf{w}_{h}\left(\mathbf{X}_{\Delta \lambda}^{(m)}\right)\right)_{0, \Lambda}$ is given by $(3.1)$, 
(ii) Compute $\mathbf{X}_{\Delta \lambda}^{(m+1)} \in \mathbf{S}_{\Delta \lambda}$ according to

$$
\mathbf{D}_{\Delta t}^{+} \mathbf{X}_{\Delta \lambda}^{(m)}=\mathbf{v}_{h}^{(m+1)}\left(\mathbf{X}_{\Delta \lambda}^{(m)}\right)
$$

Referring to $\partial B_{\Delta \lambda}^{(m)}$ as the boundary of the immersed cell at time $t_{m}$ which consists of $C^{2}$ segments $\partial B_{\Delta \lambda}^{(m, r)}$ connecting material points $\mathbf{X}_{\Delta \lambda}^{(m)}\left(\lambda_{r-1}\right)$ and $\mathbf{X}_{\Delta, \lambda}^{(m)}\left(\lambda_{r}\right), 1 \leq r \leq R$, one can deduce the estimate

$$
\left\|\nabla \mathbf{v}_{h}^{(m+1)}\right\|_{0, \partial B_{\Delta \lambda}^{(m)}}^{2} \leq C_{\text {cell }} h^{-1}\left\|\nabla \mathbf{v}_{h}^{(m+1)}\right\|_{0, \Omega}^{2}
$$

with a positive constant $C_{\text {cell }}$ depending on the triangulation $\mathcal{T}_{h}(\Omega)$ (see $(3.8)$ in [7]). A stability analysis reveals that the Backward Euler/Forward Euler FE-IB requires the CFL-type condition (cf. Theorem 3.1 in [7])

$$
\frac{\Delta t}{h} \leq \frac{\eta}{8 C_{\text {cell }}\left(\kappa_{\mathrm{e}} \Lambda_{1}+\kappa_{\mathrm{e}} \Lambda_{2}\right)},
$$

where $\Lambda_{1}$ and $\Lambda_{2}$ are given by

$$
\Lambda_{1}:=\max _{0 \leq m \leq M} \max _{\lambda \in \Lambda}\left|\frac{\partial \mathbf{X}_{\Delta \lambda}^{(m)}}{\partial \lambda}\right|, \quad \Lambda_{2}:=\max _{0 \leq m \leq M} \max _{1 \leq r \leq R}\left|\frac{\partial^{3} \mathbf{X}_{\Delta \lambda}^{(m)}}{\partial \lambda^{3}}\right|_{\Lambda_{r}} \mid .
$$

The CFL-condition (3.5) for the semi-implicit scheme means a restriction of the time-step size $\Delta t$ in particular depending on the amount of deformation of the immersed membrane as reflected by the quantities $\Lambda_{1}$ and $\Lambda_{2}$. For problems characterized by large values of $\Lambda_{1}$ and $\Lambda_{2}$, the time increments need to be chosen very small, leading to a high computational effort. As a remedy, a fully implicit time-stepping scheme can be used based on the application of the backward Euler scheme in time for both the Navier-Stokes equations and the equation of motion of the immersed boundary. This Backward Euler/Backward Euler FE-IB method is unconditionally stable at the expense that at each time-step a nonlinear algebraic system has to be solved. We refer to [10] for details including a predictor-corrector continuation strategy featuring an adaptive choice of the time-step size.

\section{Optimal control of the surface acoustic wave actuated cell sorting}

In this section, following the strategy 'discretize first, then optimize', we will formulate the optimal control problem for the surface acoustic wave actuated cell sorting. The objective is to steer the immersed cells to desired positions by controlling the electric power applied to the IDT. The semi-implicit Backward Euler/Forward Euler FE-IB method from section 3 serves as the state constraints. 
For $z:=(v, p, X)$, consider the following optimal control problem

$$
\left\{\begin{array}{rl}
\min _{z \in Z, u \in U} & J(z, u) \\
\text { s.t. } & S(z)=b(u) \\
& u \in U_{\mathrm{ad}}
\end{array}\right.
$$

The objective functional $J$ is given by

$$
J(z, u):=J\left(X_{[1]}, X_{[2]}\right):=\sum_{i=1}^{2} \frac{1}{2}\left\|\mathbf{X}_{[i], \Delta \lambda}^{(\mathcal{M}(i))}-\mathbf{X}_{[i], \Delta \lambda}^{\mathrm{des}}\right\|_{0, \Lambda}^{2},
$$

where $1 \leq i \leq 2$ are the cell indices of two different biological cells and the functions $\mathbf{X}_{[i], \Delta \lambda}^{\text {des }} \in \mathbf{S}_{\Delta \lambda}(\Lambda)$ mark desired final positions close to the respective outflow boundaries. The time instants $t_{\mathcal{M}(i)}$ are chosen such that the $\mathbf{x}_{1}$-components of barycenters of the immersed cells $\mathbf{X}_{[i], \Delta \lambda}(\Lambda, t)$ and $\mathbf{X}_{[i], \Delta \lambda}^{\text {des }}(\Lambda)$ coincide. The state operator $S$ reads

$$
S(z):=\left(\begin{array}{l}
v_{0}-v^{(0)} \\
\left(M_{E}+\Delta t A\right) v^{(m)}+\Delta t B^{\top} p^{(m)}-\Delta t f_{E}\left(X^{(m-1)}\right)-M_{E} v^{(m-1)} \\
B v^{(m)} \\
X_{0}-X^{(0)} \\
M_{L} X^{(m)}-M_{L} X^{(m-1)}-\Delta t K\left(X^{(m-1)}\right) v^{(m)}
\end{array}\right)
$$

and

$$
b(u)=\left(0, \Delta \operatorname{tg}\left(u^{(m-1)}, 0,0,0\right)^{T}, \quad 1 \leq m \leq \mathcal{M} .\right.
$$

The volume force term $g\left(u^{(m)}\right) \in \mathbb{R}^{N_{1}}$ comprises components

$$
g\left(u^{(m)}\right)_{i}:=\int_{\Omega} \mathbf{f}_{\mathrm{vol}}\left(u^{(m)}\right) \cdot \boldsymbol{\phi}_{i} \mathrm{~d} \mathbf{x}, 1 \leq i \leq N_{1},
$$

where the volume force density $\mathbf{f}_{\mathrm{vol}}$ generated by the IDT is modeled by

$$
\begin{aligned}
\mathbf{f}_{\mathrm{vol}}\left(u^{(m)}\right)(\mathbf{x}) & :=\left\{\begin{array}{ll}
\left(0, \beta u^{(m)} \mathrm{e}^{\left(-\left(\mathbf{x}_{2}-y_{0}\right) / d\right)} k\left(\mathbf{x}_{1}, x_{0}, D\right)\right)^{\top}, & \mathbf{x} \in \bar{\omega} \\
\mathbf{0} & \mathbf{x} \in \Omega \backslash \omega
\end{array},\right. \\
k\left(x, x_{0}, D\right) & =\frac{\sin ^{2}\left(2 \pi\left(x-x_{0}\right) / D\right)}{\left(2 \pi\left(x-x_{0}\right) / D\right)^{2}} .
\end{aligned}
$$

Here, $\omega \subset \Omega$ denotes the subdomain where the SAW is effective, $\beta$ stands for a transmission coefficient, $d$ for the decay length, $\left(x_{0}, y_{0}\right)^{\top}$ refers to the center position of the segment at the lower lateral boundary where the SAWs enter the domain, and $D / 2$ is the half width of this segment (marked green in Figure 2 below). The function $k$ is known as a Kirchhoff function and describes the refraction pattern of the SAW intensity.

We define the set of admissible controls by

$$
U_{\mathrm{ad}}:=\left\{u \in U:=\mathbb{R}^{\mathcal{M}} \mid u^{\min } \leq u^{(m)} \leq u^{\max }, 1 \leq m \leq \mathcal{M}\right\},
$$

where the control $u^{(m)}$ is the power applied to the IDT at time $t_{m}$ and $u^{\mathrm{min}}$, $u^{\max } \in \mathbb{R}^{\mathcal{M}}$ are given bounds. As numerical optimization scheme we use 
the well-known projected gradient with Armijo line search. To this end, we introduce the reduced objective functional

$$
J_{\text {red }}(u):=J(z(u), u),
$$

where $z(u)=(v(u), p(u), X(u))$ is the solution to $S(z)=b(u)$. Then problem (4.1) can be reformulated as the state-reduced optimal control problem

$$
\left\{\begin{aligned}
\min _{u \in \mathbb{R}^{\mathcal{M}}} & J_{\text {red }}(u) \\
\text { s.t. } & u \in U_{\text {ad }}
\end{aligned}\right.
$$

being equivalent to (4.1). Problem (4.4) can be solved by the following scheme where $\Pi_{U_{\text {ad }}}$ denotes the projection operator onto the admissible set:

Algorithm 4.1 .

(o) Let $u_{0}$ and a tolerance $\varepsilon>0$ be given.

for $\mathrm{k}=0,1,2, \ldots$

(i) Compute the descent direction $d_{k}=-\nabla j_{\text {red }}\left(u_{k}\right)$ via adjoint approach.

(ii) If $\left\|\Pi_{U_{\text {ad }}}\left(u_{k}+d_{k}\right)-u_{k}\right\|<\varepsilon$, stop: $u^{*}:=u_{k}$.

(iii) Compute a step length $\alpha_{k}$ by Armijo line search.

(iv) Update $u_{k+1}=u_{k}+\alpha_{k} d_{k}$, project it onto $U_{\text {ad }}$ and go back to (i).

The computationally most challenging part is the evaluation of $\nabla J_{\text {red }}\left(u_{k}\right)$ which is taken care of by the adjoint approach:

For the optimization problem (4.1) we consider the Lagrangian

$$
\mathcal{L}(z, u, \lambda)=j(z, u)+\langle\lambda, S(z)-b(u)\rangle_{Y^{*}, Y}, \quad \mathcal{L}: Z \times U \times Y^{*} \rightarrow \mathbb{R} .
$$

The associated state equations and adjoint state equations are

$$
\begin{aligned}
& 0=\mathcal{L}_{\lambda}^{\prime}=S(z(u))-b(u) \\
& 0=\mathcal{L}_{z}^{\prime}=J_{z}^{\prime}(z(u), u)+\left(S_{z}^{\prime}(z(u))\right)^{*} \lambda(u) .
\end{aligned}
$$

It is easy to prove the following lemma:

Lemma 4.2. Assume that $S(z)=b(u)$ has a unique solution $z(u), \forall u \in U$, and that $\lambda(u) \in Y^{*}$ is the unique solution to (4.5b). Moreover assume that the mappings $(z, u) \mapsto j(z, u), z \mapsto S(z), u \mapsto z(u)$, and $u \mapsto b(u)$ are Fréchet-differentiable. Then there holds

$$
J_{\text {red }}^{\prime}(u)=\mathcal{L}_{u}^{\prime}(z(u), u, \lambda(u)) .
$$

In more detail, one has to perform the following steps to compute the reduced gradient $\nabla J_{\text {red }}\left(u_{k}\right)$ (for notational simplicity, only one cell is considered): 
Algorithm 4.3.

(i) Compute the state $\left(v_{k}, p_{k}, X_{k}\right):=\left(v\left(u_{k}\right), p\left(u_{k}\right), X\left(u_{k}\right)\right)$ : $v_{k}^{(0)}:=v_{0}, X_{k}^{(0)}:=X_{0}$ and for $1 \leq m \leq \mathcal{M}$

$$
\begin{aligned}
\left(M_{E}+\Delta t A\right) v_{k}^{(m)}+\Delta t B^{\top} p_{k}^{(m)} & =\Delta t\left(f_{E}\left(X_{k}^{(m-1)}\right)+g\left(u_{k}^{(m-1)}\right)\right)+M_{E} v_{k}^{(m-1)} \\
B v_{k}^{(m)} & =0 \\
M_{L} X_{k}^{(m)} & =M_{L} X_{k}^{(m-1)}+\Delta t K\left(X_{k}^{(m-1)}\right) v_{k}^{(m)} .
\end{aligned}
$$

(ii) Compute the adjoint state $\left(w_{k}, q_{k}, Y_{k}\right):=\left(w\left(u_{k}\right), q\left(u_{k}\right), Y\left(u_{k}\right)\right)$ backward in time: $w_{k}^{(\mathcal{M})}:=0, Y_{k}^{(\mathcal{M})}:=X^{\text {des }}-X^{(\mathcal{M})}$ and for $\mathcal{M}-1 \geq m \geq 1$

$$
\begin{gathered}
M_{L} Y_{k}^{(m)}=M_{L} Y_{k}^{(m+1)}+\Delta t\left[f_{E}^{\prime}\left(X_{k}^{(m)}\right)^{\top} w_{k}^{(m+1)}+\left(K^{\prime}\left(X_{k}^{(m)}\right) v_{k}^{(m+1)}\right)^{\top} Y_{k}^{(m+1)}\right] . \\
\left(M_{E}+\Delta t A\right) w_{k}^{(m)}+\Delta t B^{\top} q_{k}^{(m)}=\Delta t K\left(X_{k}^{(m-1)}\right)^{\top} Y_{k}^{(m)}+M_{E} w_{k}^{(m+1)} \\
B w_{k}^{(m)}=0
\end{gathered}
$$

(iii) Set $\nabla J_{\text {red }}\left(u_{k}\right):=\partial j\left(z_{k}, u_{k}\right) / \partial u+\Delta t \sum_{m=1}^{\mathcal{M}-1}\left(w_{k}^{(m)}\right)^{\top} \nabla^{\top} g\left(u_{k}^{(m-1)}\right)$.

The derivatives showing up in the adjoint system represent the nontrivial terms of the adjoint differential operator $\left(S_{z}(z(u))\right)^{*}$ from $(4.5 \mathrm{~b})$.

Let us state the optimality conditions associated with (4.1).

Theorem 4.4 (Necessary optimality conditions). Assume the set $U_{a d}$ is given by (4.3) and the assumptions from Lemma 4.2 are fulfilled. Then there exists an optimal solution $\left(z^{*}, u^{*}\right)$ to (4.1) with associated Lagrange multiplier $\lambda^{*}$ such that: $\left(z^{*}, u^{*}\right)$ solves $(4.5 \mathrm{a}), \lambda^{*}$ solves $(4.5 \mathrm{~b})$ and

$$
\left(\nabla J_{\text {red }}\left(u^{*}\right)\right)_{i}\left\{\begin{array}{ll}
\leq 0, & u_{i}^{*}=u_{i}^{\max } \\
=0, & u_{i}^{\min }<u_{i}^{*}<u_{i}^{\max } . \\
\geq 0, & u_{i}^{*}=u_{i}^{\min }
\end{array} .\right.
$$

(4.7) can be written in short form as $\Pi_{U_{\text {ad }}}\left(u^{*}-\nabla j_{\text {red }}\left(u^{*}\right)\right)=u^{*}$. This justifies the termination criterion from Algorithm 4.1, step (ii).

\section{Numerical results}

As a numerical example, we consider the sorting scenario 'up - down', meaning that the first cell $(i=1)$ is supposed to take the upper outflow channel and the second cell $(i=2)$ the lower one.

The separation channel $\Omega$ is shown in Figure 2 featuring three inflow boundaries at the left and two outflow boundaries at the right. The main part has a length of $300 \mu \mathrm{m}$ and a width of $180 \mu \mathrm{m}$. The maximal inflow velocities $v_{\text {in }}^{\text {(left) }}, v_{\text {in }}^{\text {(top) }}$, and $v_{\text {in }}^{\text {(bottom) }}$ have been chosen according to

$$
v_{\text {in }}^{(\text {left })}=10 \mathrm{~mm} / \mathrm{s}, \quad v_{\text {in }}^{(\text {top })}=12.5 \mathrm{~mm} / \mathrm{s}, \quad v_{\text {in }}^{(\text {bottom })}=10 \mathrm{~mm} / \mathrm{s},
$$


12 T. Franke, R.H.W. Hoppe, C. Linsenmann, L. Schmid and A. Wixforth

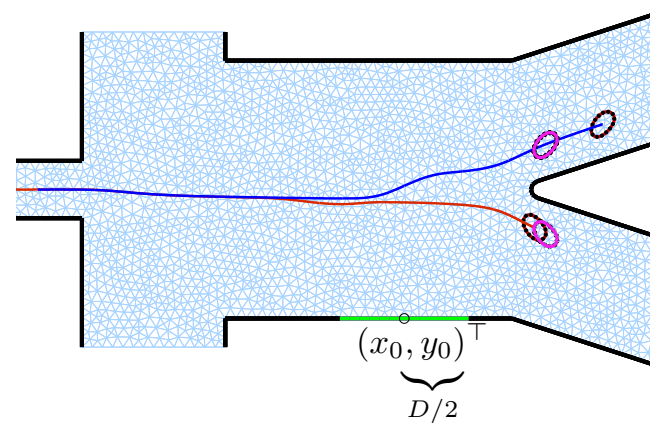

Figure 2. Paths of two different cells under the influence of the computed optimal control. The desired positions are depicted in magenta.

guaranteeing that without SAW actuation a cell leaves the channel through the lower outflow boundary. As the density $\rho$ and the dynamic viscosity $\eta$ we have chosen

$$
\rho=1000 \mathrm{~kg} / \mathrm{m}^{3}, \quad \eta=7.0 \mathrm{mPa} \cdot \mathrm{s}
$$

both for the carrier fluid and the fluid enclosed by the membrane of the two cells. We note that in practice this can be achieved using density and viscosity matching by adding suitable chemicals to the carrier fluid. We have considered initially spherical cells of diameter $16 \mu \mathrm{m}$ and moduli

$$
\kappa_{\mathrm{e}}=5.0 \cdot 10^{-5} \mathrm{~N} / \mathrm{m}, \quad \kappa_{\mathrm{b}}=1.0 \cdot 10^{-16} \mathrm{Nm} .
$$

The sorting task is complicated by setting the initial distance between the cells to $25 \mu \mathrm{m}$ only. For the spatial discretization of the Navier-Stokes equations we have used a finite element mesh with mesh size $h=7.5 \mu \mathrm{m}$, whereas for the spatial discretization of the equations of motion of the immersed boundaries we have used a partition of $\Lambda$ with $\Delta \lambda=3.6 \mu \mathrm{m}$. The time-step size $\Delta t$ in the semi-implicit Backward Euler/Forward Euler FE-IB method has been chosen according to $\Delta t=1 / 100 \mathrm{~ms}$ making sure that the CFLcondition (3.5) is satisfied.

Figure 2 shows the computed paths of the cells in the separation channel along with their designated positions at final time, whereas Figure 3 displays the computed controls during the projected gradient method.

Finally, Table 1 reflects the decrease of the reduced objective functional $J_{\text {red }}\left(u_{k}\right)$ as a function of the iteration step $k$ of the optimization algorithm.

\section{Conclusions}

We have presented an optimal control approach to the sorting of different biological cells by surface acoustic wave (SAW) manipulated fluid flow in a microfluidic separation channel. The mathematical modeling and numerical 


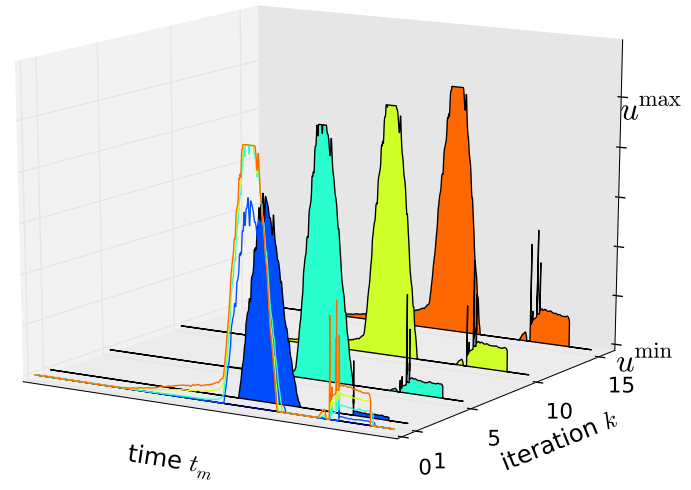

Figure 3. Evolution of the controls $u_{k}$ arising in the optimization algorithm.

TABLE 1. Decrease of the reduced objective functional $J_{\text {red }}\left(u_{k}\right)$ as a function of the iteration step $k$ of the optimization algorithm

\begin{tabular}{|r||c|c|c|c|c|}
\hline Iteration $k$ & 0 & 1 & 5 & 10 & 15 \\
\hline$J_{\text {red }}\left(u_{k}\right)$ & $8.18 \mathrm{e}+01$ & $2.53 \mathrm{e}+00$ & $1.42 \mathrm{e}+00$ & $1.09 \mathrm{e}+00$ & $9.40 \mathrm{e}-01$ \\
\hline
\end{tabular}

simulation of the fluid-structure interaction has been taken care of by the finite element immersed boundary (FE-IB) method. The feasibility of the approach has been documented by numerical results.

\section{References}

[1] D. Boffi and L. Gastaldi; A finite element approach for the immersed boundary method. Comput. Struct. 81 (2003), 491-501.

[2] D. Boffi, L. Gastaldi, and L. Heltai, Numerical stability of the finite element immersed boundary method. Math. Models and Methods in Appl. Sci. 17 (2007), 1479-1505.

[3] J.L. Carey, J.P. McCoy, and D.F. Keren, Flow Cytometry in Clinical Diagnostics. 4th Edition. ASCP Press, Chicago, 2007.

[4] M. Eisenstein, Cell sorting: divide and conquer. Nature 441 (2006), 1179-1185.

[5] T. Franke, S. Braunmüller, T. Frommelt, and A. Wixforth, Sorting of solid and soft objects in vortices driven by surface acoustic waves. Proc. SPIE, $\mathbf{7 3 6 5}$ (2009), 736500.

[6] T. Franke, S. Braunmüller, L. Schmid, and A. Wixforth, Surface acoustic wave actuated cell sorting (SAWACS). Lab Chip, 10 (2010), 789-794.

[7] T. Franke, R.H.W. Hoppe, C. Linsenmann, and K. Zeleke, Numerical simulation of surface acoustic wave actuated cell sorting. Cent. Eur. J. Math. 11 (2013), $760-778$. 
14 T. Franke, R.H.W. Hoppe, C. Linsenmann, L. Schmid and A. Wixforth

[8] T. Franke, R.H.W. Hoppe, C. Linsenmann, L. Schmid, and C. Willbold, Numerical simulation of the motion and deformation of red blood cells and vesicles in microfluidic flows. Comput. Visualiz. Sci. 14 (2011), 167-180.

[9] T.S. Hawley and R.G. Hawley, Flow Cytometry Protocols. 2nd Edition. Vol. 263, Humana Press, Totowa, NJ, 2004.

[10] R.H.W. Hoppe and C. Linsenmann, An adaptive Newton continuation strategy for the fully implicit finite element immersed boundary method. J. Comp. Phys. 231 (2012), 4676-4693.

[11] C. Peskin, Numerical analysis of flood flow in the heart. J. Comput. Phys. 25 (1977), 220-252.

[12] C. Peskin, The immersed boundary method. Acta Numerica 11 (2002), 479-517.

[13] H.M. Shapiro, Practical flow cytometry. Wiley-Liss, New York, 2003.

[14] L.A. Sklar, Flow Cytometry for Biotechnology. Oxford University Press, New York, 2005.

Thomas Franke

Institute of Physics

Universität Augsburg

D-86159 Augsburg

Germany

e-mail: franketh@physik.uni-augsburg.de

Ronald H.W. Hoppe

Institute of Mathematics

Universität Augsburg

D-86159 Augsburg

Germany

Department of Mathematics

University of Houston

Houston, TX 77204-3008

USA

e-mail: hoppe@math.uni-augsburg.de, rohop@math.uh.edu

Christopher Linsenmann

Institute of Mathematics

Universität Augsburg

D-86159 Augsburg

Germany

e-mail: christopher.linsenmann@math.uni-augsburg.de

Lothar Schmid

Institute of Physics

Universität Augsburg

D-86159 Augsburg

Germany

e-mail: lothar.schmid@physik.uni-augsburg.de 


\author{
Achim Wixforth \\ Institute of Physics \\ Universität Augsburg \\ D-86159 Augsburg \\ Germany \\ e-mail: achim.wixforth@physik.uni-augsburg.de
}

